\title{
Direct imaging of bridged twin protoplanetary disks in a young multiple star
}

\author{
Satoshi Mayama ${ }^{1}$, Motohide Tamura ${ }^{1,2}$, Tomoyuki Hanawa ${ }^{4}$, Tomoaki \\ Matsumoto ${ }^{5}$, Miki Ishii ${ }^{3}$, Tae-Soo Pyo ${ }^{3}$, Hiroshi Suto ${ }^{2}$, Takahiro \\ Naoi $^{2}$, Tomoyuki Kudo ${ }^{2}$, Jun Hashimoto ${ }^{1,2}$, Shogo Nishiyama ${ }^{6}$, \\ Masayuki Kuzuhara ${ }^{7}$ and Masahiko Hayashi ${ }^{7}$ \\ ${ }^{1}$ The Graduate University for Advanced Studies (SOKENDAI), \\ Shonan International Village, Hayama-cho, Miura-gun, Kanagawa, 240-0193, Japan \\ email: mayama_satoshi@soken.ac.jp \\ ${ }^{2}$ National Astronomical Observatory of Japan, 2-21-1, Osawa, Mitaka, Tokyo 181-8588 Japan \\ ${ }^{3}$ Subaru Telescope, National Astronomical Observatory of Japan, \\ 650 North A'ohoku Place, Hilo, Hawaii, 96720, USA \\ ${ }^{4}$ Center for Frontier Science, Chiba University, Inage-ku, Chiba, 263-8522, Japan \\ ${ }^{5}$ Faculty of Humanity and Environment, Hosei University, \\ Fujimi, Chiyoda-ku, Tokyo 102-8160, Japan \\ ${ }^{6}$ Department of Astronomy, Kyoto University, \\ Kitashirakawa-Oiwake-cho, Sakyo-ku, Kyoto, 606-8502, Japan \\ ${ }^{7}$ University of Tokyo, Hongo, Tokyo 113-0033, Japan
}

\begin{abstract}
Studies of the structure and evolution of protoplanetary disks are important for understanding star and planet formation. Here, we present the direct image of an interacting binary protoplanetary system. Both circumprimary and circumsecondary disks are resolved in the near-infrared. There is a bridge of infrared emission connecting the two disks and a long spiral arm extending from the circumprimary disk. Numerical simulations show that the bridge corresponds to gas flow and a shock wave caused by the collision of gas rotating around the primary and secondary stars. Fresh material streams along the spiral arm, consistent with the theoretical scenarios where gas is replenished from a circummultiple reservoir.
\end{abstract}

Keywords. binaries: general, stars: formation, planetary systems: protoplanetary disks

Studies of protoplanetary disks in multiple systems are essential for describing the general processes of star and planet formation because most stars form as multiples (Ghez et al. 1993). However, such circummultiple disks and spiral arms in multiple systems have rarely been directly resolved to date. Here, we present the direct image of an interacting binary protoplanetary system (Mayama et al. 2010). We investigate the geometry of a young (a stellar age of 4 million years) multiple circumstellar disk system, SR24, to understand its nature based on observations and numerical simulations. SR24 is a hierarchical multiple, located $160 \mathrm{pc}$ away in the Ophiuchus star-forming region.

We obtained an infrared image of SR24 with CIAO (Tamura et al. 2000) mounted on the Subaru 8.2-m Telescope. (Fig. 1, left). The emission arises from dust particles mixed with gas in the circumstellar structures scattering the stellar light. Both circumprimary and circumsecondary disks are clearly resolved. Both disks overflow the inner Roche lobes (dotted contours in Fig. 1), suggesting that the material outside the lobes can fall into either of the inner lobes. There is a bridge of infrared emission connecting the two disks and a long spiral arm extending from the circumprimary disk. A spiral arm would suggest that the SR24 system rotates counter clockwise. The orbital period of the binary is 


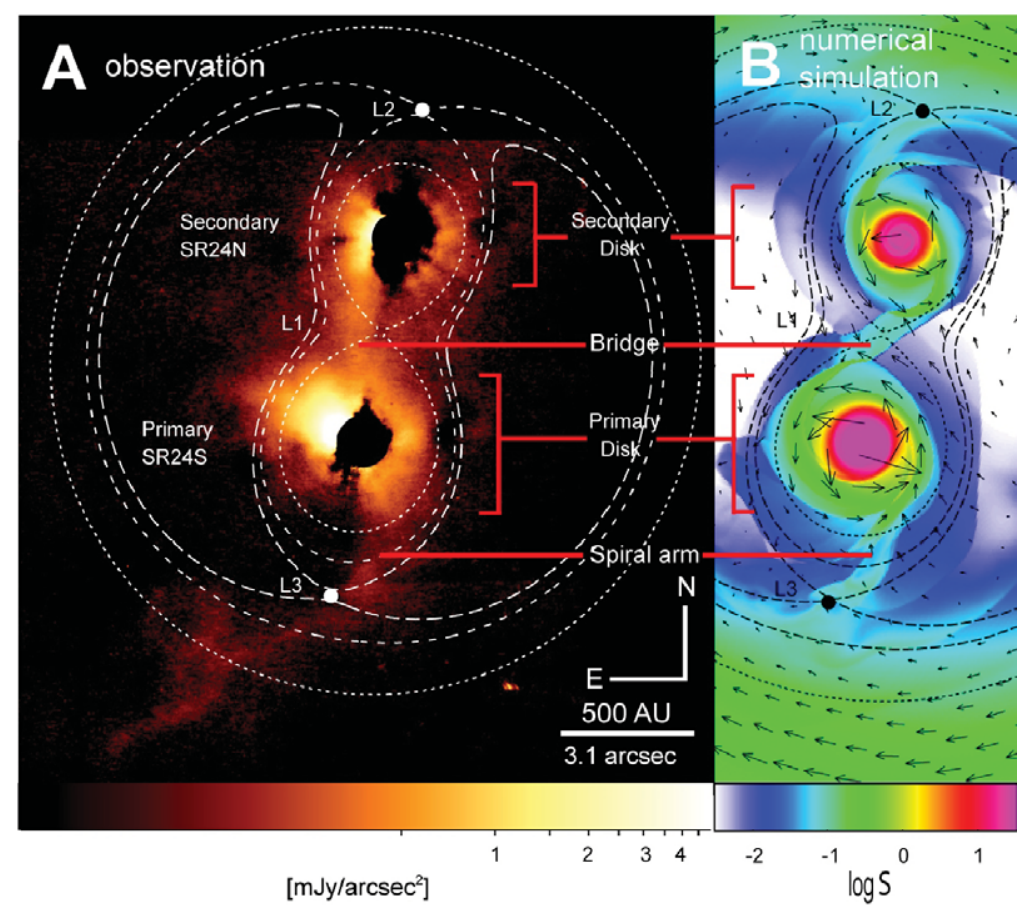

Figure 1. Observed and simulated images of the young multiple star, SR24. (A) H-band $(1.6 \mu \mathrm{m})$ coronagraphic image of SR24. The total integration time was $1008 \mathrm{~s}$. The PSFs of the final images have sizes of 0.1 arcsecond (FWHM) for the H-band. The inner and outer Roche Lobes are overlaid on the Subaru image as dotted and dashed lines, respectively. L1, L2, and L3 represent the inner Lagrangian point, outer Lagrangian point on the secondary side, and outer Lagrangian point on the primary side, respectively. (B) Snapshot of accretion onto the binary system SR24 based on 2D numerical simulations. The color and arrows denote the surface density distribution and velocity distribution, respectively.

15,000 yr. The arm would also imply replenishment of the twin disk gas from the circumbinary disk.

We performed 2D numerical simulations of accretion from a circumbinary disk to identify the features seen in the coronagraphic image(Fig. 1, right). Although the gas flow was not stationary, especially inside the Roche lobes, the stage of the $2 \mathrm{D}$ simulations shown in Fig. 1 shared common features with the observed image. These agreements between observation and simulation suggest that the bridge corresponds to gas flow and a shock wave caused by the collision of gas rotating around the primary and secondary stars. The arm corresponds to a spiral wave excited in the circumbinary disk.

\section{References}

Ghez, A. M., Neugebauer, G., \& Matthews, K. 1993, AJ, 106, 2005

Mayama, S., Tamura, M., Hanawa, T., Matsumoto, T., Ishii, M., Pyo, T. S., Suto, H., Naoi, T., Kudo, T., Hashimoto, J., Nishiyama, S., Kuzuhara, M., \& Hayashi, M. 2010, Science, 327, 306

Tamura, M., et al. 2000, Proc. SPIE, 4008, 1153 